\title{
Doppler echocardiographic evaluation of pulmonary blood flow after the Fontan operation: the role of the lungs
}

\author{
D J Penny, A N Redington
}

\begin{abstract}
Objective-To document whether the act of respiration influences pulmonary blood flow in patients after the Fontan operation.

Design-Prospective study in which patients acted as their own controls.

Setting-Supraregional paediatric cardiology centre.

Patients-Sixteen patients who had undergone atriopulmonary anastomosis (Fontan operation) for the treatment of congenital heart disease.

Intervention-Doppler assessment of pulmonary artery fow patterns with a simultaneous respirometer, electrocardiogram and phonocardiogram.

Results-Total (mean (1SD)) forward pulmonary flow was 63.6 (35)\% higher during inspiratory cardiac cycles than during expiratory ones.

Conclusions-The act of breathing provides an additional energy supply to pulmonary blood flow in patients after the Fontan operation.
\end{abstract}

Since Fontan and Baudet's description of the atriopulmonary anastomosis for the palliation of complex congenital heart disease, ${ }^{1}$ there has been considerable debate regarding the determinants of blood flow through a pulmonary vascular bed when no ventricular pumping chamber is present. For the most part this debate has centred around the importance of atrial contraction in providing an energy source for pulmonary blood flow. The potential contribution of the atrial pump to an atriopulmonary circulation was first suggested 36 years $\mathrm{ago}^{2}$ and most recently re-emphasised in this journal. ${ }^{3}$ Conversely, others have suggested that atrial activity provides no additional energy to pulmonary blood flow, ${ }^{45}$ so that the Fontan circulation has been described as one for which the sole net energy supply originates in the left ventricle. ${ }^{6}$

While the role of the right atrium in providing energy to the Fontan circulation continues to be explored, the potential role of other possible energy sources must not be forgotten. We showed that respiratory effort seems to be a powerful determinant of pulmonary blood flow in patients after total cavopulmonary connection, ${ }^{7}$ in which the right heart is completely bypassed. Furthermore, we attempted to augment the effect of normal respiration on the pulmonary circulation in these patients with negative extrathoracic pressure ventilation and we found that pulmonary blood flow increased with this technique. ${ }^{8}$ Though the potential implications of respiratory effort for pulmonary blood flow in patients after the Fontan operation have been mentioned, ${ }^{9}$ they have not been formally assessed.

We investigated whether respiratory effort influenced pulmonary blood flow in patients after the Fontan operation, in whom a subpulmonary atrium is incorporated into the pulmonary circulation.

\section{Patients and methods}

PATIENTS

Sixteen patients (11 male and five female; mean (1SD) age 119 (45.6) months) were studied 38.5 (31.5) months after atriopulmonary anastomosis. Nine patients had a preoperative diagnosis of tricuspid atresia, five had double inlet left ventricle, one had double inlet right ventricle, and one patient had double outlet right ventricle with abnormal insertion of the tricuspid valve tensor apparatus which precluded a biventricular repair. Atriopulmonary anastomosis was performed in all patients (by direct suture in eight and via an interposition homograft conduit in eight). The atrial septal defect was closed in all patients and the right atrioventricular valve orifice was closed in patients who had two atrioventricular valves.

\section{MEASUREMENTS}

Patients were studied at rest in a supine position during quiet respiration. Doppler echocardiograms were recorded from a parasternal or suprasternal position with a $5 \mathrm{MHz}$ or $3.5 \mathrm{MHz}$ transducer interfaced with a Toshiba SSH-160A ultrasound system. A pulsed wave, range gated Doppler sample volume was positioned in the pulmonary artery distal to the site of the atriopulmonary anastomosis. Simultaneous electrocardiograms, phonocardiograms, and a respirometer trace were recorded. Recordings were made at a paper speed of $100 \mathrm{~mm} / \mathrm{s}$.

\section{ANALYSIS}

Measurements were obtained over six cardiac cycles (three during inspiration and three during expiration). To separate the atrial contribution to pulmonary flow from that during the remainder of the cardiac cycle we drew a 


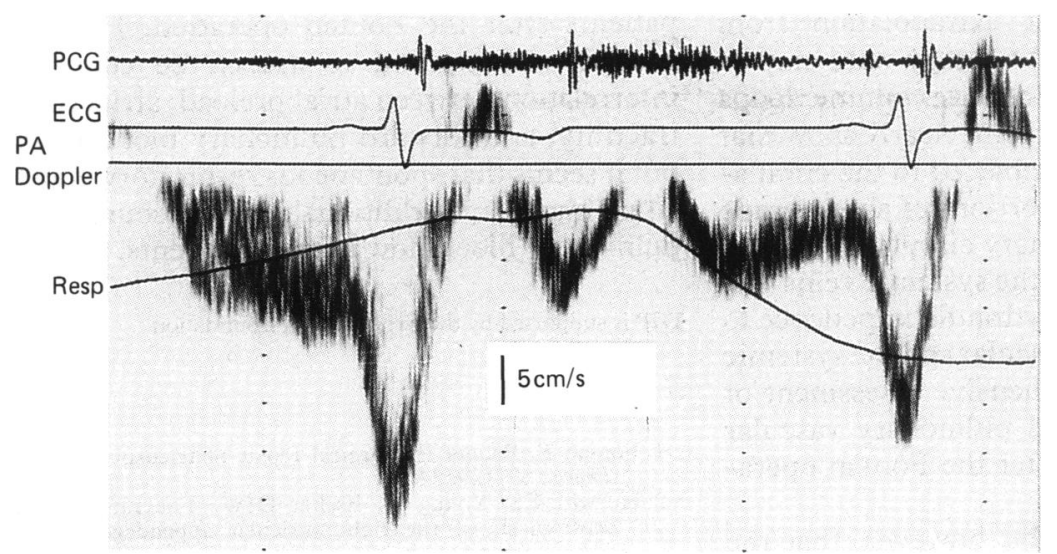

Figure Simultaneous pulmonary artery Doppler flow ( $P$ A Loppler), electrocardiogram (ECG), phonocardiogram (PCG), and respirometer (Resp) trace in a patient after the Fontan operation. During inspiration (upward deflection on the respirometer) the total forward pulmonary flow-velocity integral and peak forward flow velocity were increased. atrial systole from $39.8(16 \cdot 6) \mathrm{cm} / \mathrm{s}$ to 51.3 (19.9) $\mathrm{cm} / \mathrm{s}(\mathrm{p}<0.001)$.

\section{EFFECT OF ATRIOVENTRICULAR CONNECTION}

During expiration the atrial contribution to forward flow in the pulmonary artery tended to be higher in patients with double inlet ventricle $(57.4(6.7) \%)$ than in patients with tricuspid atresia $(48.6(8.9) \%)$, although this difference did not reach statistical significance $(p=0.059)$. Similarly, during inspiration, no significant difference in either the atrial contribution to forward flow or the peak forward flow velocity was seen in the two groups.

\section{RETROGRADE PULMONARY FLOW}

Retrograde flow was seen in the pulmonary artery during early ventricular systole in nine patients. While it was not possible to quantify the amount of retrograde flow, qualitative assessment suggested that the retrograde flow was less appreciable during inspiration.

vertical line to zero at the beginning of the onset of the waveform related to atrial systole. $^{3}$ We calculated the atrial contribution to pulmonary flow $(\%)$ as $100 \times$ the velocitytime integral during atrial contraction, divided by the velocity-time integral of total forward pulmonary flow. ${ }^{3}$ Peak forward flow velocity in the pulmonary artery was measured directly from the paper recordings.

Traces were digitised with a Summagraphics digitising plate, interfaced with a Prime 750 mainframe, so that the total pulmonary flow-velocity integral and its atrial component could be compared during inspiration and expiration. No attempt was made to calculate the area of the atriopulmonary anastomosis, which was assumed to remain constant throughout the cardiac and respiratory cycle. All indices were expressed as mean (1SD) and the statistical significance of differences between group means was assessed by Student's $t$ test. Probability values $<0.05$ were regarded as significant.

\section{Results}

PULMONARY DOPPLER FLOW

All patients were in sinus rhythm and a biphasic pattern of forward flow was seen in the pulmonary artery (fig). Low velocity forward flow occurred during ventricular systole and early diastole. During late diastole forward flow was augmented at the same time as atrial contraction, so that the atrial contribution to forward flow in the pulmonary artery during expiration was $51.4(8.8) \%$ of total forward flow.

\section{EFFECT OF RESPIRATION}

Total forward flow during inspiratory cardiac cycles was 63.6 (35)\% higher than during expiratory ones $(p<0.001)$. We saw a proportional increase in forward flow during atrial contraction, so that the contribution of atrial contraction to total flow was similar during inspiration $(52.0(10) \%)$ and expiration $(51.4(8.8) \%)(p=0.693)$ (fig).

Inspiration, furthermore, resulted in an increase in peak forward flow velocity during

\section{Discussion}

The results of this study are consistent with previously published observations of Doppler flow patterns in the pulmonary artery of patients with an atriopulmonary circulation which suggest that right atrial contraction may contribute to forward flow in the pulmonary artery. $^{310}$ The addition of simultaneous respirometry, however, provides further information about the determinants of blood flow in the atriopulmonary circulation.

It has been suggested that in the normal circulation the right atrium is at the peak of its work-function curve. ${ }^{11}$ Normal inspiration is associated with an increase in right atrial preload $^{12}$ and it thus might be expected that inspiration (especially in patients after the Fontan operation in whom the right atrium is operating further along the work-function curve than in normal subjects) would be associated with a reduction in the relative contribution of atrial contraction to pulmonary flow. Our observation that forward flow during atrial contraction is increased by inspiration, suggests that even in patients after the Fontan operation some preload reserve may be maintained by the right atrium. An alternative explanation for these data would be that during inspiration forward flow related to atrial contractility is unchanged but the inspiratory increase in pulmonary forward flow during atrial systole is a manifestation of additional passive flow with the atrium acting as a conduit during inspiration. Perhaps more important is the overall contribution that atrial contraction makes to cardiac output after this operation. Clearly, in the absence of inflow valves, atrial contraction will not only lead to forward flow in the pulmonary artery, but also to retrograde flow into the systemic veins. ${ }^{10}$ The lack of a mean pressure rise from systemic veins to pulmonary artery has been suggested as evidence against "effective" pump function of the atrium in the Fontan pulmonary circulation. ${ }^{6}$ While there can be no doubt that the atrium is an inefficient pump under these 
circumstances, a direct extrapolation from pressure measurements to energy state may be somewhat simplistic. Pressure-volume loops from the normal right atrium, clearly show that external work is being imparted to the circulation. ${ }^{12}$ The relative proportions of anterograde flow towards the pulmonary circulation and of retrograde flow towards the systemic veins will depend on the relative hydraulic impedence to flow in the pulmonary vascular and the systemic venous beds. A comprehensive assessment of atrial work-function and pulmonary vascular impedence in patients after the Fontan operation is required.

There can be no doubt, however, that the lungs are a potentially important additional source of energy to the Fontan circulation. In our patients a $64 \%$ increase in total forward pulmonary flow was seen during normal inspiration. The loss of this augmentation of flow, for example, during positive pressure ventilation, may lead to a significant reduction in cardiac output. Indeed, such a reduction has been shown during the application of low levels of positive end expiratory pressure to patients after the Fontan operation. ${ }^{13}$ We have previously shown a considerable effect of respiration on pulmonary blood flow in patients after a total cavopulmonary shunt operation, when there is no atrial chamber interposed between the systemic veins and the pulmonary artery. $^{7}$ In these patients it is possible to augment this effect and increase pulmonary blood flow by negative extrathoracic pressure. ${ }^{8}$ Based on the results of the present study one might expect a similar effect in patients after atriopulmonary circulation. The potential clinical benefits to patients requiring assisted ventilation after atriopulmonary anastomosis are obvious.

In summary, normal inspiratory effort significantly augments pulmonary blood flow in patients after the Fontan operation. Further studies are required to assess the complex interrelation between atrial preload, atrial contractility, and forward pulmonary blood flow, but it seems that spontaneous respiratory effort is an important additional energy source for pulmonary blood flow in these patients.

DJP is supported by the British Heart Foundation

1 Fontan F, Baudet E. Surgical repair of tricuspid atresia.

Thorax 1971;26:240-8.
2 Hurwitt ES, Young D, Escher DJW. The rationale of anastomosis of the right auricular appendage to the anastomosis of the right auricular appendage to the pulmonary artery in the treat
$J$ Thorac Surg 1955;30:503-12.

3 Qureshi SA, Richheimer R, McKay R, Arnold R. Doppler echocardiographic evaluation of pulmonary artery flow after modified Fontan operation: importance of atrial contraction. Br Heart J 1990;64:272-6.

4 Kreutzer GO, Vargas FJ, Schlichter AJ, et al. Atriopulmonary anastomosis. $J$ Thorac Cardiovasc Surg 1982;83 427-36.

5 de Leval MR, Kilner P, Gewillig M, Bull C. Total cavopulmonary connection: a logical alternative to atriopulmonary connection for complex Fontan operations. $J$ Thorac Cardiovasc Surg 1988;96:682-95.

6 Bull C. Atrial and ventricular dependent circulations. In: Macartney FJ, ed. Congenital heart disease. Lancaster: MTP Press, 1986:35-53.

7 Redington AN, Penny D, Shinebourne EA. Pulmonary blood flow after total cavopulmonary shunt. Br Heart $J$ 1991;65:213-7.

8 Penny DJ, Hayek Z, Redington AN. The effects of positive and negative extrathoracic pressure ventilation on pulmonary blood flow after the total cavopulmonary shun procedure. Int J Cardiol 1991;30:128-30.

9 Nakazawa $M$, Nakanishi T, Okuda $\mathrm{H}$, et al. Dynamics of right heart flow in patients after Fontan procedure. Circulation 1984;69:306-12.

$10 \mathrm{Di}$ Sessa TG, Child JS, Perloff JK, et al. Systemic venous and pulmonary arterial flow patterns after Fontan's procedure for tricuspid atresia or single ventricle. Circulation 1984 70:898-902.

11 Payne RM, Stone HL, Engleken EJ. Atrial function during volume loading. J Appl Physiol 1971;31:326-31.

12 Ferguson JJ, Miller MJ, Aroesty JM, Sahagian P, Grossman W, McKay RG. Assessment of right atrial pressurevolume relations in patients with and without an atrial volume relations in patients with and $\mathrm{A}$ Coll Cardiol 1989;13:630-6.

13 Williams DB, Kiernan PD, Metke MP, Marsh HM Danielson GK. Hemodynamic response to positive endexpiratory pressure following right atrium-pulmonary artery bypass (Fontan procedure). J Thorac Cardiovas Surg 1984;87:856-61. 